\title{
A thermoregulation model for hypothermic treatment of neonates
}

\author{
Ana Beatriz de C.G. e Silva ${ }^{\mathrm{a}, 1}$, Joanna Laszczyk ${ }^{\mathrm{b}, 1}$, Luiz C. Wrobel ${ }^{\mathrm{c}, 1, *}$, \\ Fernando L.B. Ribeiro ${ }^{\mathrm{a}, 1}$, Andrzej J. Nowak ${ }^{\mathrm{b}, 1}$ \\ ${ }^{a}$ Civil Engineering Program,COPPE/Federal University of Rio de Janeiro Technological \\ Center, Ilha do Fundão, CEP 21945-970, Rio de Janeiro, Brazil \\ ${ }^{b}$ Institute of Thermal Technology, Silesian University of Technology, Konarskiego 22, \\ 44-100 Gliwice, Poland \\ ${ }^{c}$ Institute of Materials and Manufacturing, Brunel University London, Uxbridge UB8 3PH, \\ United Kingdom
}

\begin{abstract}
This paper presents a thermoregulation finite element model (FEM) to simulate hypothermia procedures for the treatment of encephalopathy hypoxic-ischemia (EHI) in neonates, a dangerous ischemic condition that can cause neurological damages and even death. Therapeutic hypothermia is the only recommended technique to reduce sequels caused by EHI in neonates; intervention with moderate cooling for neural rescue in newborns with hypoxic-ischemic brain injury is the culmination of a series of clinical research studies spanning decades. However, the direct monitoring of brain cooling is difficult and can lead to additional tissue damage. Therefore, the measurement of efficiency during clinical trials of hypothermia treatment is still challenging. The use of computational methods can aid clinicians to observe the continuous temperature of tissues and organs during cooling procedures without the need for invasive techniques, and can thus be a valuable tool to assist clinical trials simulating different cooling options that can be used for treatment. The use of low cost methods such as cooling blankets can open the possibility of using brain cooling techniques in hospitals and
\end{abstract}

\footnotetext{
* Corresponding author

Email addresses: anabeatrizgonzaga@coc.ufrj.br (Ana Beatriz de C.G. e Silva), joanna.jachowicz@gmail.com (Joanna Laszczyk), luiz.wrobel@brunel.ac.uk (Luiz C. Wrobel ), fernando@coc.ufrj.br (Fernando L.B. Ribeiro ), andrzej.j.nowak@polsl.pl (Andrzej J. Nowak )

${ }^{1}$ Tel.: $+44(0) 1895266696$
}

Preprint submitted to Journal of LATEX Templates

April 14, 2016 
clinics that cannot currently afford the available expensive equipment and techniques. In this work, we developed a FEM package using isoparametric linear three-dimensional elements which is applied to the solution of the continuum bioheat Pennes equation. Blood temperature changes were considered using a blood pool approach. The results of the FEM model were compared to those obtained through the implementation of a user-defined function (UDF) in the commercial finite volume software FLUENT and validated with experimental tests. Numerical analyses were performed using a three-dimensional mesh based on a complex geometry obtained from MRI scan medical images.

Keywords: thermoregulation, hypothermia, brain cooling, neonates, finite element method

Word Count: 4993

\section{Introduction}

2 Although the use of hypothermia as a therapeutic treatment refers to the 3 Ancient Greece [1, only in the last century the effects of hypothermia on metabolism were better comprehended, allowing its use in the global cooling of the human body. In the last decades, the positive effects of mild hypothermia after cardiac arrest and brain trauma were observed, and only very recently these effects were studied for applications post-trauma. It has been shown that therapeutic hypothermia can minimize sequels caused by hypoxic-ischemic conditions resulting of insufficient perfusion in tissues. In this condition, the brain is the most vulnerable tissue [1, and the first few hours after the ischemia are the critical time when secondary factors such as hypotension, hypoxia, hyperglycaemia and hyperthermia may occur and cause brain cell damage [2].

In neonates, hypothermia is the only known treatment for encephalopathy hypoxic-ischemic (EHI), a dangerous ischemic condition that can cause neurological damages and even death. EHI is usually a consequence of complications 
during birth such as suffocation by umbilical cord, ingestion of amniotic fluid and placenta displacement. After a consistent record of neuroprotection in animal research, induced hypothermia was investigated in several clinical trials with neonates suffering perinatal asphyxia [3].

In 2006, Edwards and Azzopardi [4] discussed extensive experimental data resulting from clinical trials for the CoolCap project [5], from the National Institute of Child Health and Human Development (NICHD) [6] and from Eicher et al. 3], and concluded that:" either selective head cooling or total body cooling reduces the combined chance of death or disability after birth asphyxia. However, as there are still unanswered questions about these treatments, many may still feel that further data are needed before healthcare policy can be changed to make cooling the standard of care for all babies with suspected birth asphyxia."

A database review between 1993 and 2008 [7] shows that many authors agreed that brain cooling is a promising therapeutic treatment in reducing brain damage in neonates. Eight randomised controlled trials 8] comprising 638 infants with moderate/severe encephalopathy showed a statistically significant reduction in mortality and neurodevelopmental disability in neonates after treatment.

Extensive experimental and clinical research carried out into prolonged moderate hypothermia for perinatal asphyxial encephalopathy was recently reported by Azzopardi et al. [9]. They also report data from the UK TOBY Cooling Register, which was set up immediately following the conclusion of enrolment to the TOBY (Total Body Hypothermia) trial, a multicentre randomised controlled trial of whole body hypothermia for the treatment of perinatal asphyxial encephalopathy, predominantly carried out in the UK. The TOBY Cooling Register already contains data available from 1331 reported cases.

Gluckman et al. [5] stated that a mild hypothermia treatment in neonates, at least six hours after detection of the hypoxic condition, is associated with positive neurological and physiological outcomes. Furthermore, the duration and intensity of cooling can determine the effects of the treatment in reducing damages [10]. The treatment consists in a reduction of the core temperature to 
$33-34^{\circ} \mathrm{C}$ for 48 to 72 hours and a rewarming phase at a rate of approximately $0.5^{\circ} \mathrm{C}$ per hour.

Hypothermic treatment in neonates can be performed by different methods. The most widely used are selective brain cooling, which consists of a cooling helmet/cap or a pack of ice placed in the head to reduce temperature, as used in the CoolCap trials, and whole body cooling, that uses a cooling blanket to decrease the core temperature of the body, as used in the TOBY trial. Although clinical trials have shown that the hypothermic treatment reduces the sequels of perinatal asphyxia, the efficacy of the different cooling methods is hard to measure through clinical trials. Gluckman et al. [5] suggest that the first method can allow effective brain cooling to be achieved with less systemic hypothermia, reducing the adverse systemic effects of the cooling. On the other hand, clinical trials suggest that whole body cooling results in better outcome in severe EHI cases, in which selective brain cooling would not be protective [11.

Proper evaluation of the cooling procedures requires that the deep brain temperatures,where cell loss leads to the most severe long term neurological impairments, are to be measured [12. Not only is the brain temperature difficult to measure but also, in the case of neonates, their immunological system is more fragile and the body is much more susceptible to temperature changes than adults. As their brain is still under development, the vulnerabilities and healing potential are different to that of an adult [2]. Although ischemia damages in neonates are much similar to those observed in adults, factors such as the duration of the treatment and the goal temperature may vary [10].

With the advances in the development of computational methods, the use of numerical modelling to simulate diseases and biological conditions in the human body has become an important tool to aid clinicians and researchers to understand the processes. Advances in computer modelling allow a detailed analysis of all information collected from the patient, the study of the influence of various parameters, facilitate the interpretation of the diagnosis, and enable the construction of models of a specific pathological condition and their use as a prognostic tool during treatment. 
Heat transfer in the human body can be affected by several mechanisms such as environmental conditions, thermophysical properties of tissues and fluids, vascular geometry, physiological changes and pathologies [13. Bioheat models describe the heat flux in the human body and have an important role in understanding heat transfer in human tissues. These models are usually analyzed on a macro-scale considering a continuum media composed by a mix of blood and tissues, as is the case of the Pennes model used in this work 14. The Pennes model is one of the most popular bioheat models and assumes that heat transfer in the tissues occurs only at the capillary vessels [15. It describes the bioheat transfer in a simple way and it was shown to be very efficient for different bioheat applications [16].

The main goal of the hypothermia treatment is to reduce the temperature in the brain. Studies simulating hypothermia in the human head purely based on bioheat models showed that, because of the influence of arterial temperature in the tissue temperature, the temperature inside the brain is not affected by external cooling [17, unless an invasive procedure such as intracarotide saline infusion is applied [18, 19. In Zhu and Diao [20] and Ley and Bayazitoglu [21, simulations of hypothermia procedures considering only the head were not effective in reducing the deep brain temperature. As the arterial temperature is responsible for regulating local tissue temperature, protecting the tissues against external cooling, the model employed to simulate hypothermia in the body must be able to take into account arterial temperature changes [22, 23].

For this reason, in this paper, we adopted a model that considers thermoregulation responses as the body tries to recover the heat loss and re-establish the homoeothermic balance, reducing the efficiency of the treatment 24]. In the last decades, several models were developed to try to reproduce the thermoregulation system, from simple two-node models to more complex multi-segment models [25, 26]. In Schwarz et al. [27], an 128 segment hemodynamic model developed by Avolio 28] is used as an input for a thermoregulation model based on Fiala [29] for hypothermia simulations during open heart surgery. Other examples of applications have been found in different fields, such as the automotive 
industry, environmental comfort and biomedical engineering [30].

The model presented in this paper was implemented in a finite element software developed at the Structures and Materials Laboratory at the Federal University of Rio de Janeiro. The idea of segments and a central blood pool is adapted from Fiala 29], but implemented here as part of a three-dimensional model which assumes that all segments are connected and exchange heat with neighbouring segments. The simulation results were compared to those presented in Laszczyk and Nowak [31, and were also validated with some available experimental results.

\section{Methodology}

\subsection{Bioheat Transfer}

The transport of blood in the tissue is a difficult process to be modeled at the microscopic level due to the large amount of vessels present in the tissues. In this paper, we considered a continuum macro-scale model based on blood perfusion developed by Pennes [13. The model considers blood and tissue as a continuous homogeneous medium. The Pennes' equation is given by

$$
\rho_{t} c_{t} \frac{\partial T_{t}}{\partial t}=\nabla \cdot\left(k_{t} \Delta T_{t}\right)+\rho_{b} c_{b} \omega_{b}\left(T_{a}-T_{t}\right)+\dot{q}_{m}
$$

and represents the bioheat flux in a domain $\Omega$. In the above equation, $T$ is the temperature and the subscripts $t, b, a$ and $m$ represent tissue, blood, arterial blood and metabolism, respectively. The material properties defined in the equation are: $k$ (thermal conductivity), $c$ (specific heat), $\rho$ (density) and $\omega$ (blood perfusion rate). The metabolic heat generation rate is represented by $\dot{q}_{m}$. The perfusion term and the metabolic heat generation rate are considered as isotropic heat sources. The arterial temperature $T_{a}$ is obtained considering the heat exchanges during blood circulation in the body, and will be discussed in the next section.

The bioheat equation is defined in all parts of the body considering different properties for each tissue. The boundary conditions are prescribed temperatures 
$\bar{T}\left(\Gamma_{t} . t\right)$ in the boundary $\Gamma_{t}$ and heat fluxes $\bar{q}\left(\Gamma_{q}, t\right)$ in the boundary $\Gamma_{q}, \Gamma=$ $\Gamma_{t} \cup \Gamma_{q}$. The initial condition is,

$$
T\left(x, t_{0}\right)=T_{0},
$$

where $T_{0}$ is the initial temperature in the tissue.

\subsection{Metabolism}

The metabolic heat generation rate in a specific tissue can be considered as a composition of the basal rate $\dot{q}_{m, 0}$, representing a thermal neutrality condition, and an additional rate $\Delta \dot{q}_{m}$ generated by a local thermoregulation activity:

$$
\dot{q}_{m}=\dot{q}_{m, 0}+\Delta \dot{q}_{m}
$$

The additional rate $\Delta \dot{q}_{m}$ can be divided into three components:

$$
\Delta \dot{q}_{m}=\Delta \dot{q}_{m, 0}+\Delta \dot{q}_{m, s h}+\Delta \dot{q}_{m, w}
$$

where $\Delta \dot{q}_{m, 0}$ refers to local basal metabolic variation and $\Delta \dot{q}_{m, s h}, \Delta \dot{q}_{m, w}$ are variations due to changes in metabolism caused by shivering and muscular effort, present only in muscular tissues. The local basal metabolic variation occurs in muscular and non-muscular tissues, and reflects the dependence of the biochemical reactions on the local temperature of the tissue. It results of the van't Hoff $Q_{10}$ effect 32 , and can be calculated by the equation:

$$
\Delta \dot{q}_{m, 0}=\dot{q}_{m, 0}\left[Q_{10}^{\frac{T_{t}-T_{0}}{10}}-1\right]
$$

In the above equation, the reference temperature $T_{0}$ is the equilibrium temperature of the body and the $Q_{10}$ coefficient is usually considered as equal to 2 . The second and third terms in Eq. (4), $\Delta \dot{q}_{m, s h}$ and $\Delta \dot{q}_{m, w}$, were not considered in the case of neonates presented in this paper. 


\subsection{Blood Circulatory System}

The perfusion term in Eq. (1) considers that the heat exchange between blood and tissues occurs only on the capillary vessels, neglecting the heat exchange between adjacent arteries and veins in the body extremities, where the blood is colder than in the core. To consider these effects, the arterial temperature calculation is obtained using the circulatory system model developed by Fiala [29], assuming a non-uniform distribution of arterial temperature in the human body.

The main hypothesis of this circulatory model is that a central blood pool supplies the tissues through the main arteries. A counter current heat exchange between adjacent arteries and veins occurs before the blood reaches the capillary vessels. In this heat exchange, the arteries lose heat and the veins are rewarmed while flowing back to the central blood pool. The venous blood from the whole body is gathered in the blood pool and a new arterial temperature is obtained.

The arterial temperature is calculated according to the following equation:

$$
T_{a}=\frac{\rho_{b} c_{b} \int \omega_{b} d V T_{c}+h_{x} T_{v 0}}{\rho_{b} c_{b} \int \omega_{b} d V+h_{x}}
$$

The symbol $T_{c}$ stands for the blood pool temperature, $T_{a}$ and $T_{v}$ are the arterial and venous temperature and $h_{x}$ is the counter current heat exchange coefficient, considered as zero in the core and with defined values for the extremities of the body 32 .

As described in Fiala et al. 25], the blood pool temperature $T_{c}$ is assumed to be a function of the tissue temperature in the whole body. Differently from Fiala [29], the implementation presented here is a three-dimensional model which considers that all sectors of the body are smoothly connected and heat is exchanged across all surfaces of each segment. The numerical procedure to calculate $T_{a}$ and $T_{c}$ will be shown in Section 2.6. 
186

\subsection{Blood Perfusion Rate}

The blood perfusion rate $\omega_{b, t}$ in a specific tissue can be divided into two components:

$$
\omega_{b, t}=\omega_{b, 0, t}+\Delta \omega_{b, t}
$$

where $\omega_{b, 0, t}$ is the local basal blood perfusion rate and $\Delta \omega_{b, t}$ is a local variation depending on the tissue temperature, calculated as:

$$
\Delta \omega_{b, t}=\Delta \omega_{b, 0, t}\left[Q_{10}^{\frac{T_{t}-T_{0}}{10}}-1\right]
$$

\subsection{External Heat Exchange}

The heat exchange between the body and the surrounding environment can be divided into two main mechanisms: convection and radiation. The heat exchange rate varies along the body surface, and the heat flux is the sum of the contributions of both convective and radiative fluxes:

$$
q_{s k i n}=q_{c o n v}+q_{r a d}
$$

The convective flux $q_{\text {conv }}$ between the skin surface and the external environment can be calculated using the Newton cooling law, defined as

$$
q_{c o n v}=h_{c o n v}\left(T_{e x t}-T_{s k i n}\right)
$$

The symbols $T_{e x t}$ and $h_{\text {conv }}$ indicate the external temperature and the heat transfer coefficient, respectively.

The radiative flux between the skin and the surrounding environment can be obtained by the Stefan-Boltzmann law:

$$
q_{\text {rad }}=h_{\text {rad }}\left(T_{\text {skin }}^{4}-T_{\text {sr,mean }}^{4}\right)
$$


where $T_{\text {skin }}$ is the temperature at the skin surface, $T_{s r, m e a n}$ is the mean temperature of the surrounding radiating surfaces and

$$
h_{\text {rad }}=\sigma \varepsilon
$$

in which $\sigma$ refers to the Stefan-Boltzmann constant and $\varepsilon$ is the average emissivity of all radiating surfaces. The emissivity may vary depending on the surface material (skin, clothes, hair).

\subsection{Numerical Model}

In the numerical model, the finite element method is used to obtain an approximate solution to the Pennes equation and the blood pool model described in Fiala et al. 25]. We have developed an in-house finite element code to solve the proposed bioheat/blood pool model. This code was designed for parallel distributed/shared memory architectures and can be run in any platform with multi-core technology. An in-house model gives more flexibility in choosing specific time operators, nonlinear algorithms, iterative solvers, control parameters and special data structures for high performance computing, as shown in Ribeiro e nd Ferreira 33] and Ribeiro and Coutinho [34.

To solve the transient problem, a time-marching scheme based on a semidiscrete form of the FEM is used, where the spatial discretization is performed by finite elements and the time derivative is approximated by finite difference operators using the two-point closed Newton-Cotes formula, also called Trapezoidal rule. Considering the Pennes Eq. (1) in a spatial domain $\Omega$ and a temporal interval $(0, \Pi)$, the domain $\Omega$ is discretized in elements and at each time step $t=t_{n+1}$ we adopt the approximation:

$$
T\left(x, t_{n+1}\right) \cong \tilde{T}\left(x, t_{n+1}\right)=\sum_{j=1}^{k} N_{j}(x) \tilde{T}_{j, n+1}
$$

where $N_{j}(x)$ refers to the spatial interpolation functions and $\tilde{T}_{j, n+1}$ are the nodal values of the approximate temperature function $\tilde{T}$ at time step $t_{n+1}$. The 
time derivative of $T$ at $t_{n+1}$ is approximated by

$$
\left.\frac{\partial T}{\partial t}\right|_{t=t_{n+1}} \cong \tilde{\dot{T}}(x)=\sum_{j=1}^{k} N_{j}(x) \tilde{\dot{T}}_{j, n+1}
$$

Introducing the approximations presented above, the following system of algebraic equations is obtained at time $t=t_{n+1}$ :

$$
M \dot{T}_{n+1}+K T_{n+1}=F_{n+1}
$$

where $M$ is the mass matrix, $\dot{T}_{n+1}$ are the nodal values of the time derivative of temperature, $K$ is the stiffness matrix, $T_{n+1}$ are the nodal temperatures at time step $t_{n+1}$ and $F_{n+1}$ is the vector of independent terms. The coefficients of these matrices are calculated as follows:

$$
m_{i j}=\int_{\Omega} c_{t} \rho_{t} N_{i} N_{j} d \Omega
$$

$$
k_{i j}=k \int_{\Omega}\left(\frac{\partial N_{i}}{\partial x} \frac{\partial N_{j}}{\partial x}+\frac{\partial N_{i}}{\partial y} \frac{\partial N_{j}}{\partial y}+\frac{\partial N_{i}}{\partial z} \frac{\partial N_{j}}{\partial z}\right) d \Omega+\int_{\Omega} c_{a} \rho_{a} \omega_{a} N_{i} N_{j} d \Omega
$$

$$
f_{i}=\int_{\Omega} q_{m} N_{i} d \Omega-\int_{\Gamma} \bar{q} N_{i} d \Gamma+\int_{\Omega} c_{a} \rho_{a} \omega_{a} T_{a} N_{i} d \Omega
$$

The counter current heat exchange and the circulatory system effects on blood pool temperature are incorporated by calculating the arterial temperature in each sector $T_{a, k}$ as

$$
T_{a, k}=\frac{\rho_{b} c_{b}\left(\sum_{i=1}^{N_{k}} \omega_{b, t, k} V_{i, t, k}\right) T_{c}+h_{x, k} T_{v 0, k}}{\rho_{b} c_{b}\left(\sum_{i=1}^{N_{k}} \omega_{b, t, k} V_{i, t, k}\right)+h_{x, k}}
$$

where the subscript $k$ denotes the sector of the body, $N_{k}$ is the number of elements in each sector $k$ and $V_{i, t, k}$ is the volume of element $i$ of the tissue $t$ in the sector $k$. The blood pool temperature used in Eq. 19 is given by Laszczyk and Nowak 31$]$ : 


$$
=\frac{\sum_{k=1}^{K}\left[\frac{\rho_{b} c_{b}\left(\sum_{i=1}^{N_{k}} \omega_{b, t, k} V_{i, t, k}\right)\left(\sum_{i=1}^{N_{k}} V_{i, t, k} T_{i, t, k}\right)}{\rho_{b} c_{b}\left(\sum_{i=1}^{N_{k}} \omega_{b, t, k} V_{i, t, k}\right)+h_{x, k}}\right]}{\sum_{k=1}^{K}\left[\frac{\left[\rho_{b} c_{b}\left(\sum_{i=1}^{N_{k}} \omega_{b, t, k} V_{i, t, k}\right)\right]^{2}}{\rho_{b} c_{b}\left(\sum_{i=1}^{N_{k}} \omega_{b, t, k} V_{i, t, k}\right)+h_{x, k}}\right]}
$$

In the above equation, $K$ is the total number of sectors in the body and $T_{i, t, k}$ represents the temperature of each element $i$ of tissue $t$ in sector $k$. In this way, at each new iteration of the system, before assembling the stiffness matrix and the force vector, the temperatures $T_{a, k}, T_{c}$ and $T_{v 0, k}$ must be updated.

A predictor multi-corrector algorithm is used for the treatment of the nonlinearities [35. For this purpose, the residue at time $t_{n+1}$ is considered as equal to zero if the system is balanced, i.e.

$$
R=F_{n+1}-M \dot{T}_{n+1}-K T_{n+1}
$$

with $R$ being the residue at time step $t_{n+1}$. In this work, we implemented the algorithm presented by Ribeiro and Ferreira [33, as illustrated in Table 1 . where $\Delta t$ is the time step and $\alpha$ is a finite difference coefficient. The system of equations is assembled by performing a local calculation in each element and it is solved using the conjugate gradient method with a diagonal pre-conditioner [36] consisting of the diagonal coefficients of the system matrix. 
Table 1: Predictor/ Multi-corrector algorithm 33

$\left.\begin{array}{lll}1: & \tilde{T}_{n+1}=T_{n}+(1-\alpha) \Delta \tilde{T}_{n} & \text { (predictor) } \\ \hline 2: & i=0 & \\ \hline 3: & T_{n+1}^{i}=\tilde{T}_{n+1} & \\ \hline 4: & \dot{T}_{n+1}^{i}=0 & \\ \hline 5: & R^{i}=F_{n+1}-M \dot{T}_{n+1}^{i}-K T_{n+1}^{i} \\ 6: & \Delta \dot{T}_{n+1}^{i+1}=\left(M^{*}\right)^{-1} R^{i}(1) \\ 7: & \dot{T}_{n+1}^{i+1}=\dot{T}_{n+1}^{i}+\Delta \dot{T}_{n+1}^{i+1} \\ 8: & T_{n+1}^{i+1}=\tilde{T}_{n+1}+\alpha \Delta t \dot{T}_{n+1}^{i+1} \\ 9: & i=i+1 \\ (1) & M^{*}=M+\alpha \Delta t\end{array}\right\}$ (corrector loop)

\section{Application}

The main purpose of this work is to implement a thermoregulation model capable of simulating the heat transfer in the human body during hypothermic treatments in neonates suffering from encephalopathy hypoxic-ischemia.

The geometrical model used in this work was obtained by segmentation of 3D medical images provided by the Institute of Thermal Technology of the Silesian Institute of Technology. It represents a neonate weighting $3.3 \mathrm{~kg}$, whose geometry consists of nine different materials (skin/fat, muscle, bone, brain, viscera, lungs, eyes, fontanel and cerebrospinal fluid) divided in seven sectors (trunk, head, abdomen, arm, hand, leg, foot). Only half of the body was used in the simulation, due to symmetry. The geometry of the neonate and the materials can be seen in Figures 1 - 2. The mesh was discretized in 3.5 million four-node tetrahedral elements. 


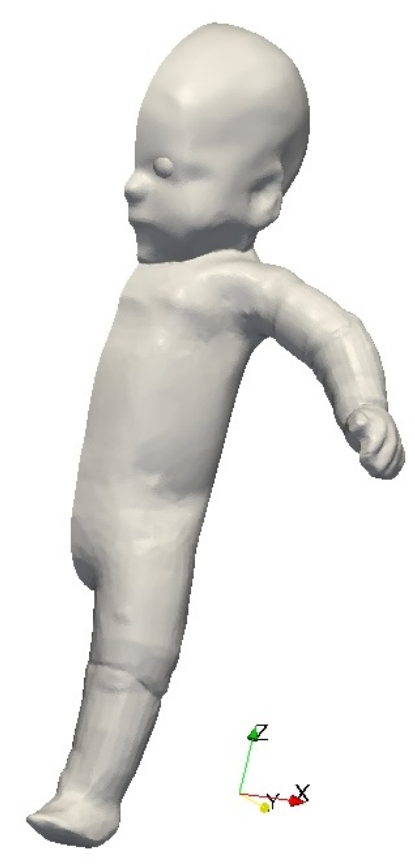

(a) External boundaries

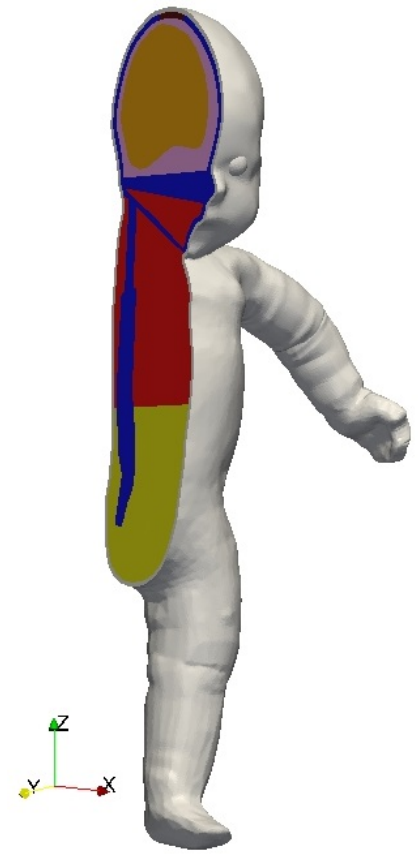

(b) Internal organs

Figure 1: Geometry of the neonate 


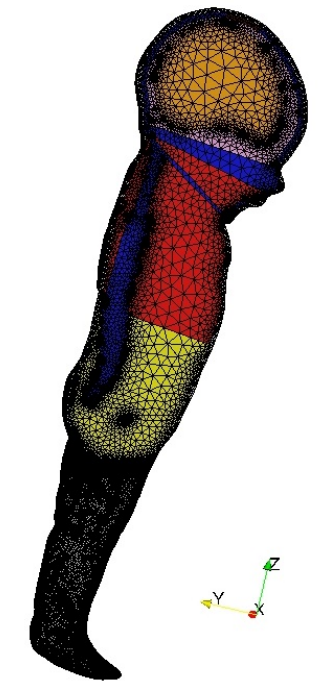

(a) Mesh of 3.5 million elements

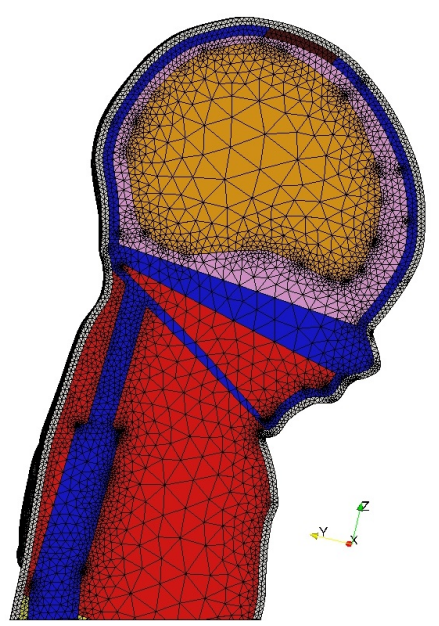

(b) Zoom -Mesh of 3.5 million elements

Figure 2: Mesh of 3.5 million elements

The material properties were taken from Laszczyk and Nowak [31]. The blood perfusion and heat generation rates were taken from an inverse analysis study [37. Table 2 shows the material properties for each tissue used in this simulation. 
Table 2: Material properties of the different tissues

\begin{tabular}{|c|c|c|c|c|c|}
\hline \multicolumn{6}{|c|}{ Material properties } \\
\hline Tissue & $\begin{array}{l}\text { Thermal } \\
\text { Con- } \\
\text { ductivity } \\
\left(W / m .{ }^{\circ} \mathrm{C}\right)\end{array}$ & $\begin{array}{l}\text { Density } \\
\left(\mathrm{kg} / \mathrm{m}^{3}\right)\end{array}$ & $\begin{array}{l}\text { Specific Heat } \\
\left(\mathrm{J} / \mathrm{kg} \cdot{ }^{\circ} \mathrm{C}\right)\end{array}$ & $\begin{array}{l}\text { Metabolic } \\
\text { Heat Gener- } \\
\text { ation Rate } \\
\left(\mathrm{W} / \mathrm{m}^{3}\right)\end{array}$ & $\begin{array}{ll}\text { Blood } & \text { Per- } \\
\text { fusion } & \text { Rate } \\
(1 / s) & \end{array}$ \\
\hline Blood & 0.5 & 1050 & 3800 & - & - \\
\hline Eye & 0.6 & 1000 & 3990 & 0 & 0 \\
\hline Lungs & 0.4 & 700 & 3719 & 850 & 0.39016 \\
\hline Skin + Fat & 0.3 & 1000 & 3631 & 450 & 0.00795 \\
\hline $\begin{array}{l}\text { Cerebrospinal } \\
\text { fluid }\end{array}$ & 0.5 & 998 & 3800 & 0 & 0 \\
\hline Bones & 0.8 & 1030 & 1796 & 0 & 0 \\
\hline Fontanel & 0.6 & 1015 & 2714 & 0 & 06 \\
\hline Muscle & 0.5 & 1000 & 3645 & 350 & 0.00135 \\
\hline Viscera & 0.5 & 1005 & 3697 & 4020 & 0.004326 \\
\hline Brain & 0.5 & 1000 & 3805 & 6800 & 0.017453 \\
\hline
\end{tabular}

Several examples were performed to initially validate the finite element model, and their results compared to those obtained through the implementation of the model in a commercial finite volume software (FLUENT) using a user-defined function (UDF). The examples were taken from an experimental study with an anthropomorphic thermal manikin of a newborn baby 38. From the experiments, the convection heat transfer coefficient was defined and the mean skin temperature was used to compare experimental and numerical results.

In the first test, the body of the neonate was divided in two regions: top and bottom. An adiabatic condition was prescribed at the bottom surface while, 


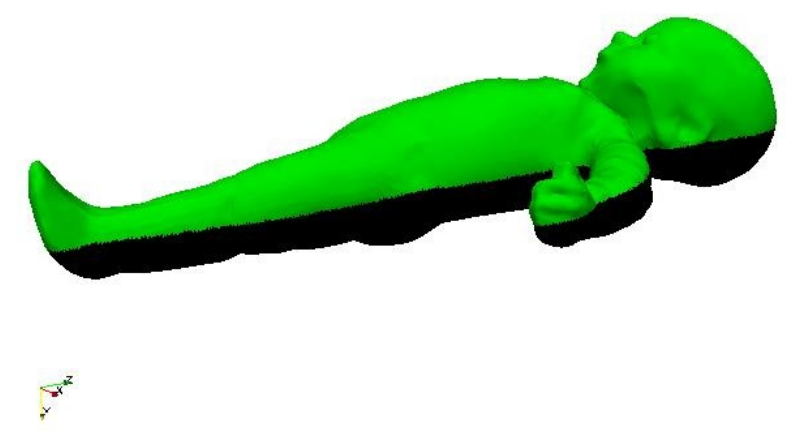

Figure 3: Top (green) and bottom (black) surfaces used to prescribe boundary conditions in case 1

The first case considers an external temperature of $30^{\circ} \mathrm{C}$ and a convective heat transfer coefficient of $10.63 \mathrm{~W} / \mathrm{m}^{2} \cdot{ }^{\circ} \mathrm{C}$. The total basal metabolic heat source was considered as $5.5 \mathrm{~W}$ and the case was analyzed as steady-state. The counter current heat exchange coefficients are presented in Table 3 . Based on these initial boundary condition values, different sub-cases are analyzed to compare both finite element and Fluent UDF implementations. An analysis with heat transfer coefficient of $9.3 \mathrm{~W} / \mathrm{m}^{2} .{ }^{\circ} \mathrm{C}$ is performed to compare the numerical results with the temperature measures of the manikin test. Finally, a sensitivity analysis is performed to analyze the behavior of the body temperature under different external conditions and heat transfer coefficients.

Table 3: Counter current heat exchange coefficient of the seven sectors of the neonate body

\begin{tabular}{ccccccccc}
\multicolumn{8}{c}{ Countercurrent heat exchange coefficient $-h_{x c}\left(W /{ }^{\circ} C\right)$} \\
\hline Sector & Head & Trunk & Abdomen & Arm & Hand & Leg & Foot \\
\hline & 0.000 & 0.000 & 0.000 & 1.652 & 0.228 & 2.760 & 1.360 \\
\hline
\end{tabular}

For the second case, a transient cooling treatment procedure was simulated. 
The treatment consists of a special cooling helmet to provide selective brain cooling while maintaining core temperature at safe levels using a radiant warmer. The goal of the hypothermic treatment is to maintain a core temperature of $33-34^{\circ} \mathrm{C}$ during 72 hours. The cooling helmet was imitated using a convective boundary condition with an external temperature of $10.8^{\circ} \mathrm{C}$ and a convective heat transfer coefficient of $19.5 \mathrm{~W} / \mathrm{m}^{2} .{ }^{\circ} \mathrm{C}$. In the rest of the body, a convective condition with external temperature of $25^{\circ} \mathrm{C}$ and convective heat transfer coefficient of $5.0 \mathrm{~W} / \mathrm{m}^{2} .{ }^{\circ} \mathrm{C}$ was prescribed during the first hour of simulation. After that, a mixed condition of convective and radiative heat flux is prescribed at the top part of the body, considering the average temperature of all radiant surfaces as $48^{\circ} \mathrm{C}$ and an average emissivity of 0.98 . The boundaries indicating the cooling helmet, the top and the bottom part are shown in Figure 4.

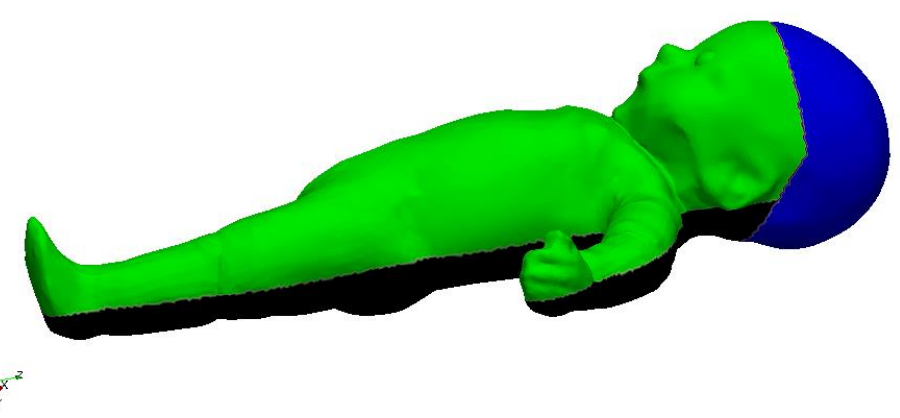

Figure 4: Cooling helmet (blue), top surface (green) and bottom surface (black) used to prescribe boundary conditions in case 2

A third case consisting of the same boundary condition of case 2 is analyzed, changing the radiative flux boundary condition to be applied during the whole simulation. The comparison between both cases is discussed at the end of the next section.

The rewarming phase is simulated in the fourth case. In this case, the cooling helmet is turned off and a controlled rewarming is performed until the normal core temperature of $37^{\circ} \mathrm{C}$ is achieved. As specified in Gluckman et al. [5], the rewarming procedure takes about $4-6$ hours and the average temperature of all 
radiant surfaces is considered to be $35-37^{\circ} \mathrm{C}$, at a maximum temperature increase rate of $0.5^{\circ} \mathrm{C} /$ hour. In the present case, the radiant warmer temperature was set as $37^{\circ} \mathrm{C}$ and the rewarming was simulated during 5.5 hours.

\section{Results}

The first test described in the previous section consists of a steady-state problem with external temperature prescribed at the top part of the body. This example was used to compare the results obtained using the finite element model and the FLUENT implementation of the thermoregulation model. Several subcases were simulated considering different conditions presented in Table 4 . Table 5 shows the blood pool temperature used to compare the results of both methods.

Table 4: Subcases considered in Case 1.

\begin{tabular}{|c|c|c|c|c|}
\hline Subcase & $\begin{array}{l}\text { Heat transfer coef- } \\
\text { ficient }\left(W / m^{2} \cdot{ }^{o} C\right)\end{array}$ & $\begin{array}{l}\text { Metabolic heat } \\
\text { generation rate } \\
\text { and blood per- } \\
\text { fusion rate }\end{array}$ & $\begin{array}{l}\text { Blood Pool } \\
\text { approach }\end{array}$ & $\begin{array}{l}\text { Division into } \\
\text { sectors }\end{array}$ \\
\hline $\mathrm{a}$ & 10.6 & constant & no & no \\
\hline $\mathrm{b}$ & 10.6 & constant & yes & no \\
\hline $\mathrm{c}$ & 10.6 & Eq. (5), Eq. 8 & yes & no \\
\hline d & 10.6 & constant & yes & yes \\
\hline $\mathrm{e}$ & 10.6 & Eq. (5), Eq. (8) & yes & yes \\
\hline $\mathrm{f}$ & 9.3 & Eq. $(5 p$, Eq. $(8)$ & yes & yes \\
\hline
\end{tabular}


Table 5: Comparison between FEM model and Fluent UDF implementation

\begin{tabular}{cccc}
\hline \multicolumn{4}{c}{$T_{p}\left({ }^{\circ} \mathrm{C}\right)$} \\
\hline Case & FEM Bioheat program & Fluent implementarion & \% difference \\
\hline a & 37.00 & 37.00 & $0.00 \%$ \\
b & 34.81 & 34.87 & $0.17 \%$ \\
c & 34.01 & 34.06 & $0.15 \%$ \\
d & 34.96 & 35.02 & $0.17 \%$ \\
e & 34.87 & 34.92 & $0.14 \%$ \\
f & 35.21 & 35.27 & $0.17 \%$ \\
\hline
\end{tabular}

323

The maximum relative difference in Table 5 is about $0.17 \%$, which means the results of both programs are in good agreement. In the case 1e, the change in the heat transfer coefficient compensates a small difference between the surface area of the experimental manikin and the geometrical model used in the simulation. The change in the heat transfer coefficient provides a mean top surface temperature of $34.5^{\circ} \mathrm{C}$, compatible with the experimental results obtained by Laszczyk and Nowak [39] using the manikin. The sensitivity of the analysis to the heat transfer coefficient and external temperature was checked by performing some analyses using different external temperatures and heat transfer coefficient values of $100 \%, 50 \%$ and $25 \%$ of the initial value for a constant external temperature. The results are shown in Table 6 
Table 6: Sensitivity study of the blood temperature to the external temperature and heat transfer coefficient.

\begin{tabular}{ccc}
\hline External Temperature $\left({ }^{\circ} \mathrm{C}\right)$ & Heat transfer coefficient $\left(\mathrm{W} / \mathrm{m}^{2} .{ }^{\circ} \mathrm{C}\right)$ & $T_{p}\left({ }^{\circ} \mathrm{C}\right)$ \\
\hline 30 & 10.63 & 34.87 \\
20 & 10.63 & 24.81 \\
10 & 10.63 & 14.80 \\
10 & 5.315 & 19.21 \\
10 & 2.6575 & 33.80 \\
\hline
\end{tabular}

A drop of $10^{\circ} \mathrm{C}$ in the external temperature results in a final blood pool temperature approximately $10^{\circ} \mathrm{C}$ lower. The relation between heat transfer coefficient and blood pool temperature is inversely proportional. Considering an external temperature of $10^{\circ} \mathrm{C}$, a heat transfer coefficient of half and a quarter of the initial value results in an increase of almost $5^{\circ} \mathrm{C}$ and $19^{\circ} \mathrm{C}$ in the blood pool temperature, respectively. These results are consistent with the physics in that, the more clothes are worn, the lower the heat transfer coefficient and less heat is lost to the environment, so the body is kept warmer.

Our aim is for some of the more sensitive parameters to be 'learned' from further studies on neonates. For instance, our collaborators at the Silesian University of Technology have already performed some inverse analyses where specific parameters were identified from temperature measurements provided by a collaborating hospital [40. As more studies on neonates are published, we hope that the required model parameters will be refined and their values will be more clearly defined.

The second test consists of a transient thermal analysis of a $3.3 \mathrm{~kg}$ neonate laying down in a mattress during 24 hours (considering the heat flux between the neonate body and the mattress to be zero), wearing a cooling helmet and subjected to a radiative flux after one hour of the beginning of the cooling procedure. The blood pool temperature during the 24 hours simulations can be 
depicted in Figure 5 .

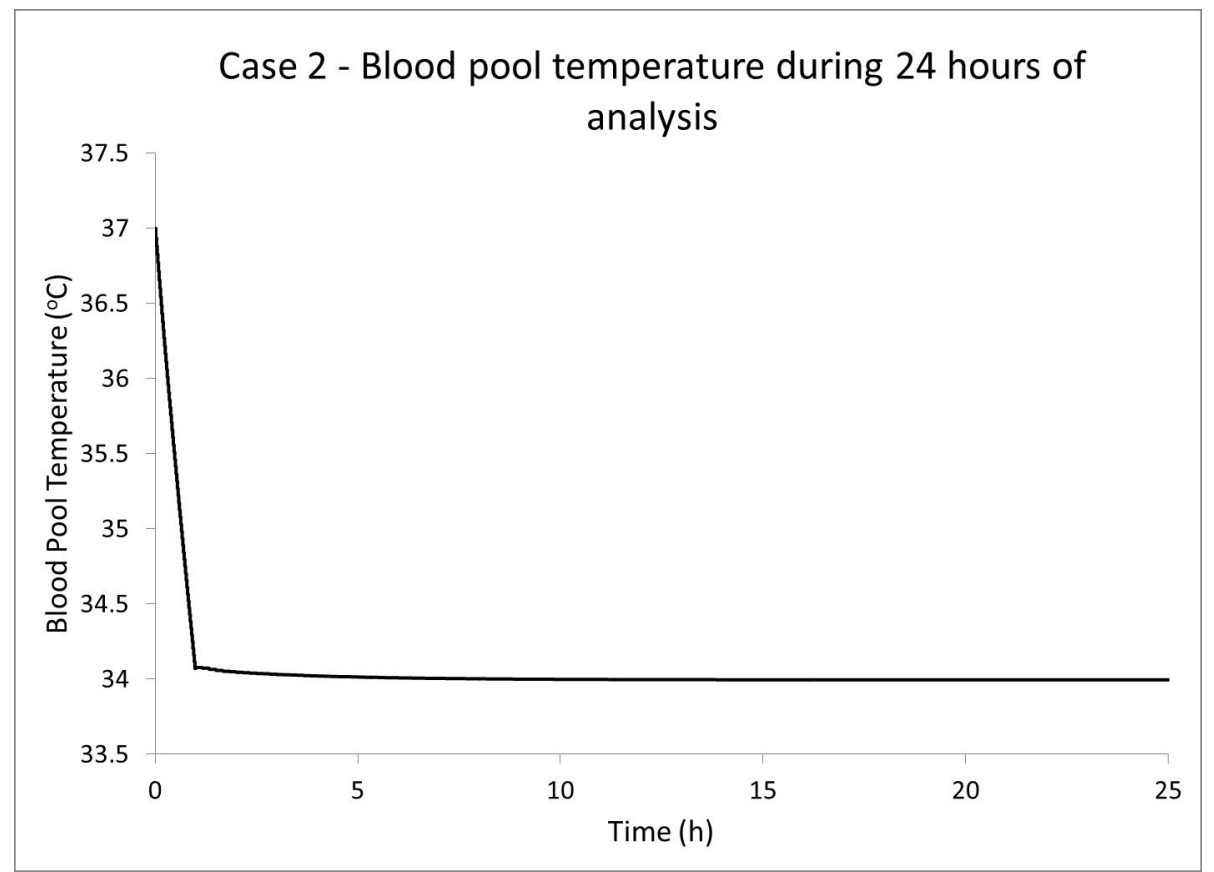

Figure 5: Case 2- Blood pool temperature during 24 hours of analysis

Figure 5 shows a blood pool temperature drop of $3{ }^{\circ} \mathrm{C}$ during the first hour of simulation, and then the maintenance of a mild hypothermic temperature of $34^{\circ} \mathrm{C}$ during the remaining 23 hours. The deep brain temperature reached $34.1^{\circ} \mathrm{C}$ after one hour of simulation, which is compatible with the hypothermia treatment temperature described in the literature. Figures 6 and 7 show the temperature profile at the skin surface and inside the body during the final hour of analysis. 


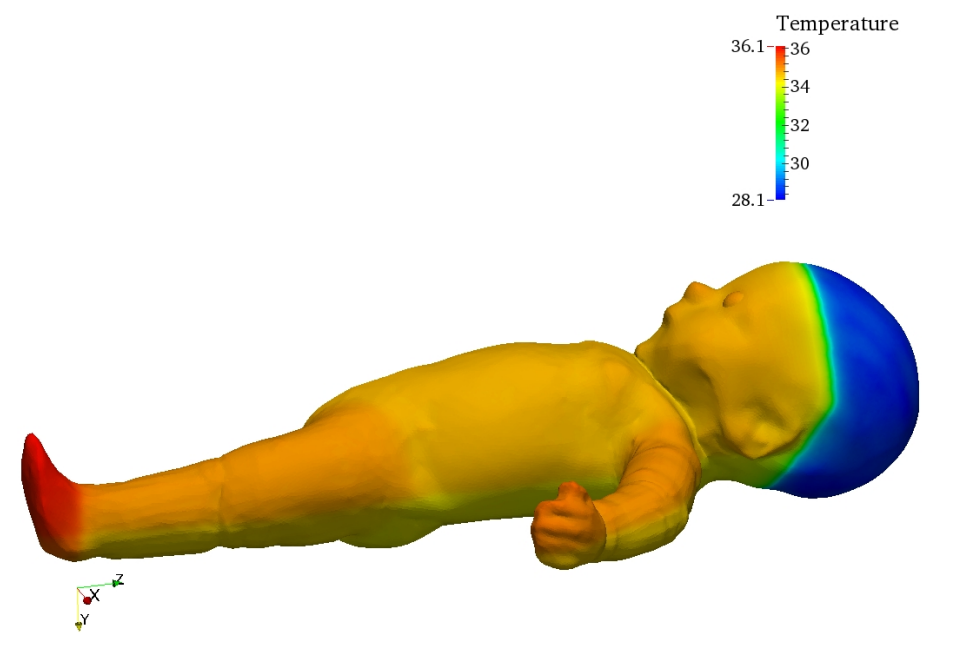

Figure 6: Case 2- Temperature profile on the skin surface after 24 hours of analysis

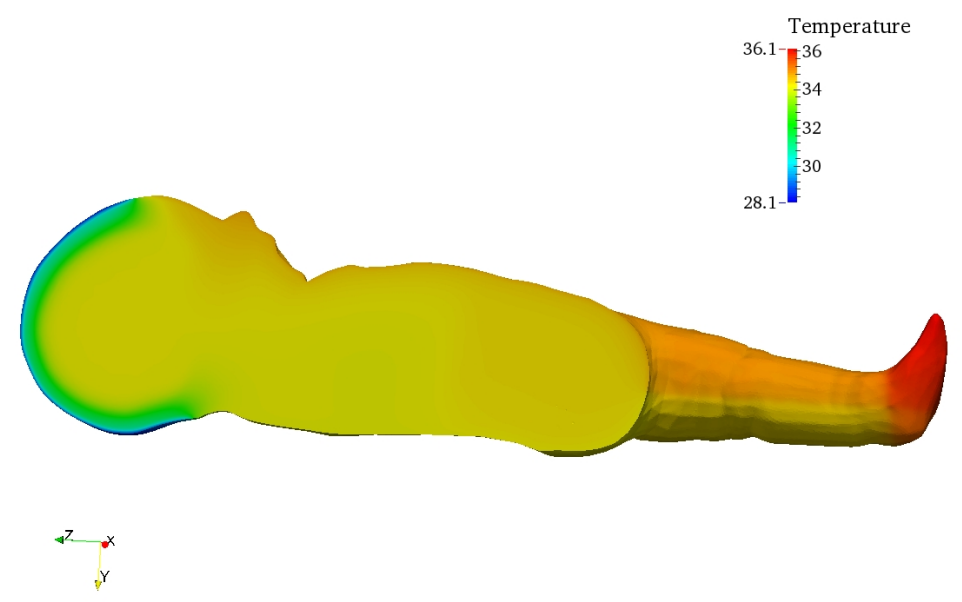

Figure 7: Case 2- Temperature profile in the interior of the body after 24 hours of analysis

The temperature at the skin surface reached a maximum of $36.1^{\circ} \mathrm{C}$ in the extremities and a minimum of $28.1^{\circ} \mathrm{C}$ at the cooling helmet location. The mean top skin temperature is $34.8^{\circ} \mathrm{C}$. The third test considers a mixed convective and 


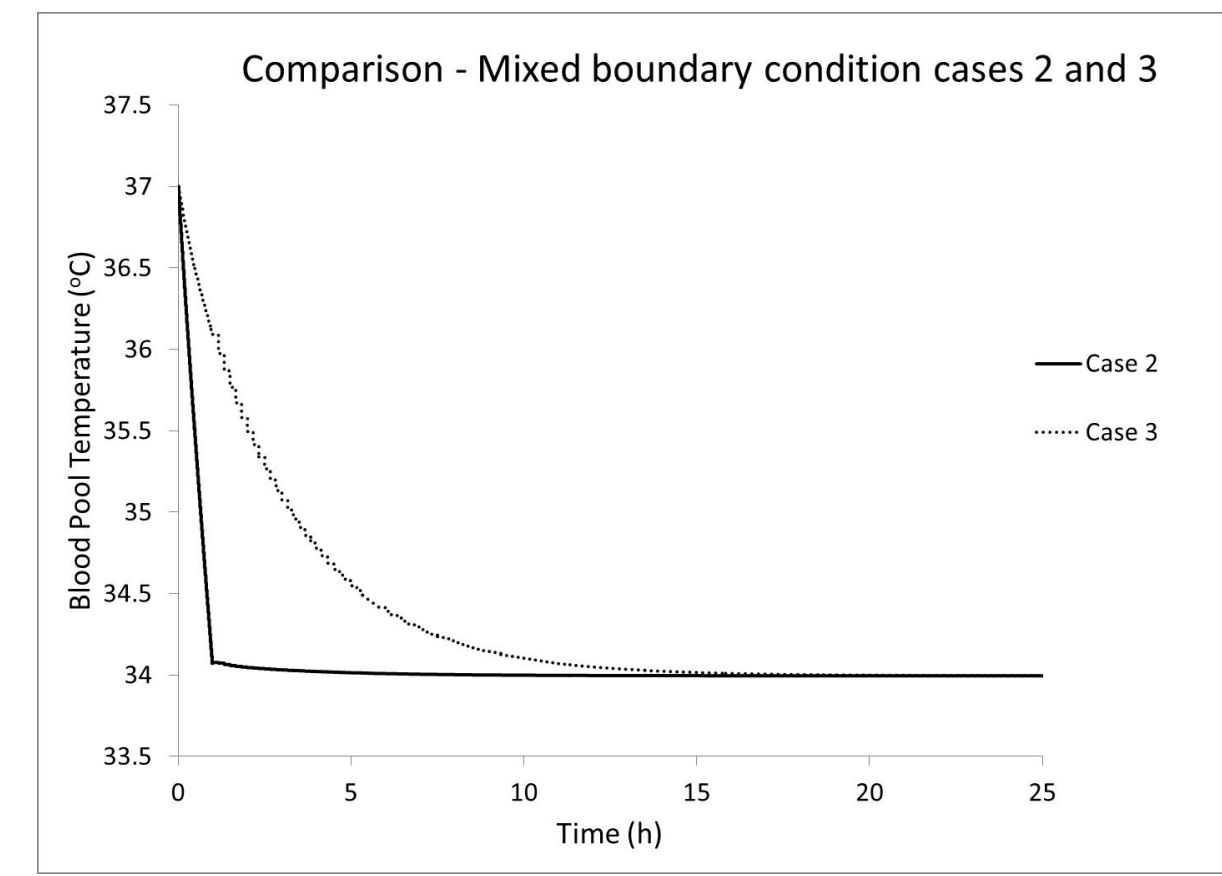

radiative flux condition during the whole analysis. A comparison between the blood pool temperature during the 24 hour simulations of the second and third cases is shown in Figure 8 .

Figure 8: Comparison between blood pool temperature in cases 2 and 3 during 24 hours of analysis

The temperature variation in the transient analysis of the third case shows that it takes about 15 hours for the blood pool temperature to drop to $34^{\circ} \mathrm{C}$ considering a radiant heat source during the whole analysis, while it takes only one hour if the radiant source is turned off during the first hour of simulation. A faster cooling process is more effective in preventing neurological damages resulting from perinatal asphyxia in neonates. For that reason, the second case would be more suitable for the cooling therapy treatment. The fourth case has considered the rewarming phase of the hypothermic treatment, with the cooling helmet turned off and the radiant warmer temperature adjusted to $37^{\circ} \mathrm{C}$. The 
results are depicted in Figure 9 .

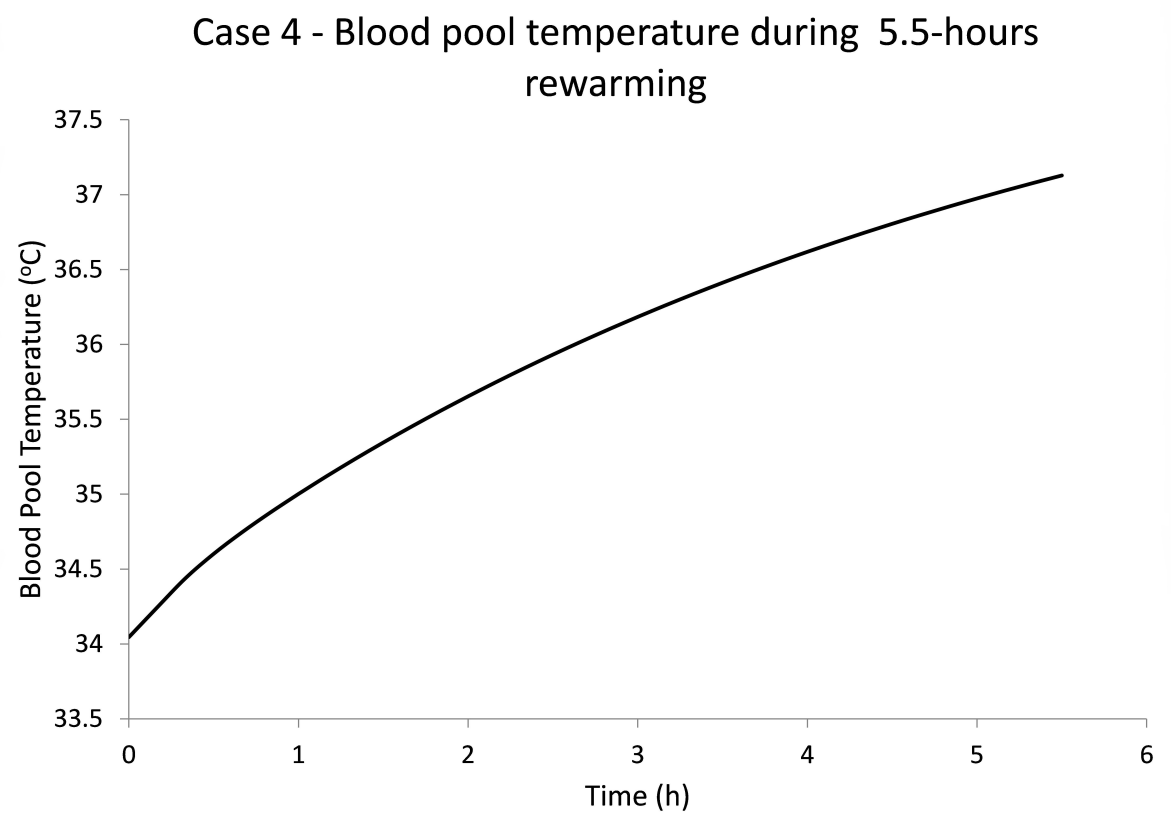

Figure 9: Case 4: Blood pool temperature during 5.5 hours analysis of rewarming procedure

The results of the fourth case simulation show that it takes about 5.5 hours to re-establish the normal core temperature of $37^{\circ} \mathrm{C}$ after a period of mild hypothermic treatment. The behaviour of the core temperature during a 24 hour hypothermic treatment is shown in Figure 10. 


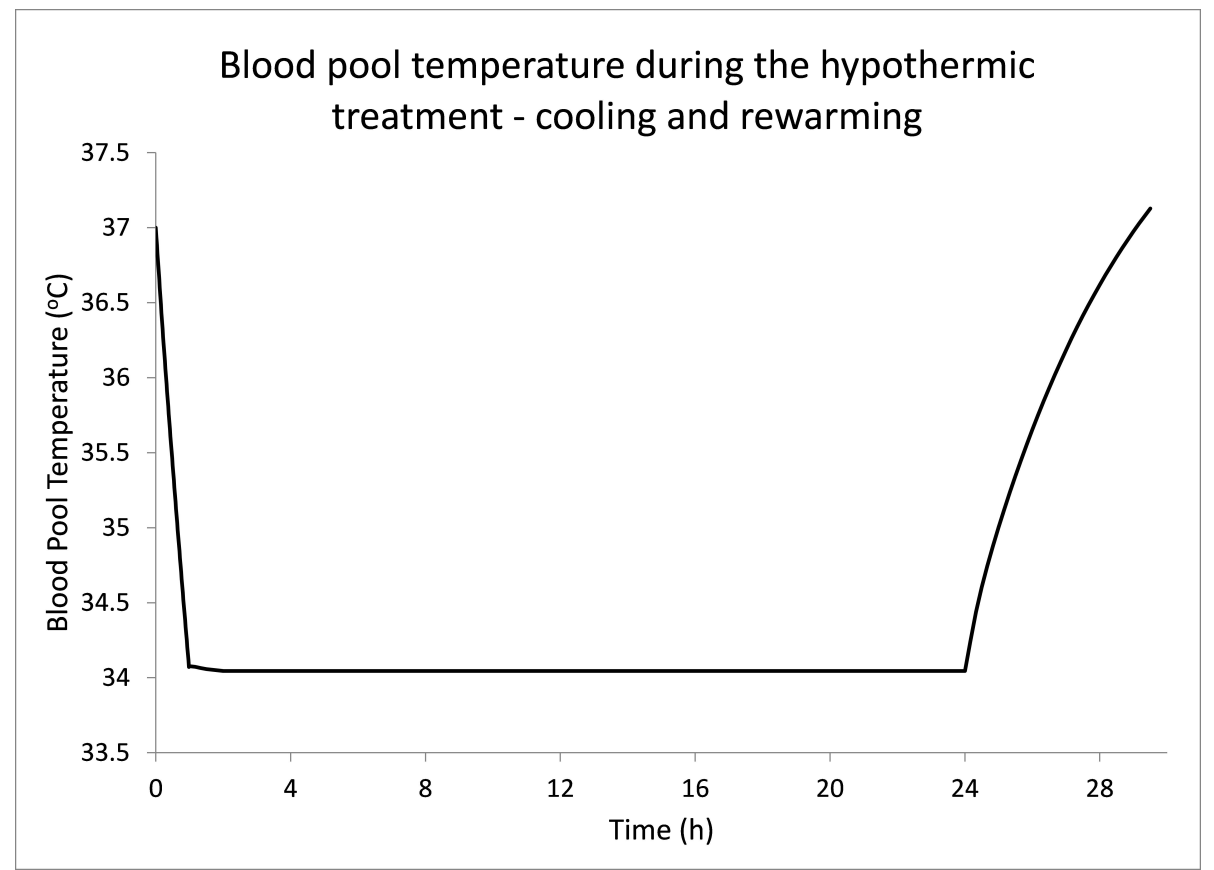

Figure 10: The blood pool temperature during the hypothermic treatment simulation, consisting of a 1 hour cooling stage, 23 hours of mild hypothermia and 5.5 hours rewarming

\section{Conclusion}

The main goal of the work described in this paper is to develop a finite element model able to simulate bioheat transfer processes in a neonate's body, and to perform selective body cooling procedures as a treatment for encephalopathy hypoxic-ischemic disease.

The results produced by the FEM code were initially compared with those obtained by a UDF Fluent implementation to validate the model and assess the differences between both methods of implementation. Results showed very small differences between both methods, concluding they are in good agreement.

A sensitivity analysis was performed to verify the dependence of the final result on the environmental temperature and heat transfer coefficient of the external surfaces. Changes in the environmental temperature show a linear 
dependence between the final temperature of the blood pool and the external temperature. On the other hand, the blood pool temperature has an inversely proportional dependence with the heat transfer coefficient, and the relation is more complex. For a $10^{\circ} \mathrm{C}$ external temperature, for example, dropping the heat transfer coefficient by a quarter resulted in an increase in the blood temperature of almost $20^{\circ} \mathrm{C}$, showing the importance of correctly determining this parameter.

After calibrating the heat transfer coefficient to compensate some differences in the surface area between the numerical geometry and a manikin developed at the Silesian University of Technology, a simulation was performed and the mean top surface temperature calculated to compare with the temperature measurements in the manikin experiment, also producing good agreement.

Finally, a transient analysis was performed considering a cooling helmet and a radiative flux on the top surface of the body. The variation of the blood pool temperature and the internal brain temperature were calculated during 24 hours. Results show it is possible to decrease the brain temperature to $34.3^{\circ} \mathrm{C}$ in one hour and to maintain it constant for 24 hours by turning on the radiant warmer after 1 hour from the start of the treatment. If the radiant warmer is turned on at the start of the treatment, it takes about 15 hours for the brain to reach the mild hypothermic temperature required in the treatment.

During the rewarming phase, after turning off the cooling helmet, it took about 5.5 hours for the normal temperature of $37^{\circ} \mathrm{C}$ to be re-established, at a rate of approximately $0.5^{\circ} \mathrm{C} /$ hour. During a selective body cooling treatment, it usually takes between 4 and 6 hours for the temperature of $37^{\circ} \mathrm{C}$ to be reached during the rewarming phase, depending on the hypothermic temperature prescribed. This means the results obtained have good correspondence with clinical results using known selective cooling devices, in the sense they correctly reproduce all the main features demonstrated in clinical trials of hypothermic treatments. .

These results demonstrate the importance of the model developed in this work for the study of different scenarios of cooling procedures. The model presented here was capable to reproduce a hypothermic treatment procedure in a 
realistic neonate body under different scenarios with very good results. This opens the way for the optimisation of the treatment on a patient-specific basis, by performing parametric studies with newborns of different weights/gestation periods to verify if the current cooling/rewarming guidance is optimal for all newborns, or if the brain temperature and rewarming period depend on the size of each newborn. The greatest difficulty is the determination of the correct parameters to guarantee that the analysis corresponds to the real case, as small differences in some of the parameters can result in substantial differences in temperature, as demonstrated by the sensitivity analysis.

Acknowledgements: We thank the Carlos Chagas Filho Research Foundation of the State of Rio de Janeiro (FAPERJ) and the Coordination for the Improvement of Higher Education Personnel (CAPES), both in Brazil, for the financial support.

\section{References}

[1] S. A. Tisherman, F. Sterz, Therapeutic Hypothermia, Springer, San Francisco, 2005.

[2] R. W. Hickey, M. J.Painter, Brain injury from cardiac arrest in children, Neurologic Clinics 24 (2006) 147-158.

[3] D. J. Eicher, C. L. Wagner, L. P. Katikaneni, T. C. Hulsey, W. T. Bass, D. A. Kaufman, M. J. Horgan, S. Languani, J. J. Bhatia, L. M. Givelichian, K. Sankaran, J. Y. Yager, Moderate hypothermia in neonatal encephalopathy: efficacy outcomes, Pediatric Neurology 32 (2005) 11-17.

[4] A. D. Edwards, D. V. Azzopardi, Therapeutic hypothermia following perinatal asphyxia, Archives of Disease in Childhood Fetal and Neonatal Edition 91 (2006) F127-F131.

[5] P. D. Gluckman, J. S. Wyatt, D. Azzopardi, R. Ballard, A. D. Edwards, D. M. Ferriero, R. A. Polin, C. M. Robertson, M. Thoresen, A. Whitelaw, A. J. Gunn, Selective head cooling with mild systemic hypothermia after 
neonatal encephalopathy: multicentre randomised trial, The Lancet 365 (2005) 663-670.

[6] S. Shankaran, A. Laptook, R. A. Ehrenkranz, Whole body hyperthermia for neonates with hypoxic ischemic encephalopathy, New England Journal of Medicine 353 (2005) 1574-1584.

[7] A. S. Araujo, S. S. Pacheco, A. G. Oliveira, C. Imaizumi, L. C. Abreu, Hypothermy as a protective strategy in asphyxiated newborns after hypoxicischemic encephalopathy, Rev. Bras. Crescimento Desenvolvimento Humano 18 (3) (2008) 346-358.

[8] S. E. Jacobs, R. Hunt, W. O. Tarnow-Mordi, T. E. Inder, P. G. Davis, Cooling for newborns with hypoxic ischaemic encephalopathy, Cochrane Database of Systematic Reviews 20074.

[9] D. Azzopardi, B. Strohm, L. Linsell, A. Hobson, E. Juszczak, J. J. Kurinczuk, P. Brocklehurst, A. D. Edwards, Implementation and conduct of therapeutic hypothermia for perinatal asphyxial encephalopathy in the UK, Analysis of national data, PLOS ONE 7.

[10] F. Coubourne, D. Corbett, Delayed and prolonged post-ischemic hypothermia is neuroprotective in the gerbil, Brain Research 654 (2) (1994) 265-272.

[11] S. Shankaran, Neonatal encephalopathy: treatment with hypothermia, Journal of Neurotrauma 26 (2009) 437-443.

[12] J. W. Han, G. M. V. Leeuwen, S. Mizushina, J. B. V. de Kamer, K. Maruyama, T. Sugiura, D. V. Azzopardi, A. D. Edwards, Monitoring of deep brain temperature in infants using multi-frequency microwave radiometry and thermal modelling, Physics in medicine and biology 46 (2) (2001) 1885-1903.

[13] A. Bhowmik, R. Singh, R. Repaka, S. Mishra, Conventional and newly developed bioheat transport models in vascularized tissues: A review, Journal of Thermal Biology 38 (3) (2013) 107-125.

xxix 
[14] J. Fan, L. Wang, A general bioheat model at macroscale, International Journal of Heat and Mass Transfer 54 (1-3) (2011) 722-726.

[15] C. Diao, L. Zhu, H. Wang, Cooling and rewarming for brain ischemia or injury: Theoretical analysis, Annals of Biomedical Engineering 31 (3) (2003) 346-353.

[16] G. M. V. Leeuwen, J. W. Hand, J. J. Lagendijk, D. V. Azzopardi, A. D. Edwards, Numerical modeling of temperature distributions within the neonatal head, Pedriatric Research 48 (3) (2000) 351-356.

[17] D. A. Nelson, S. A. Nunneley, Brain temperature and limits on transcranial cooling in humans: quantitative modeling results, Journal of Applied Physiology 78 (4) (1998) 353-359.

[18] A. A. Konstas, M. A. Neimark, A. F. Laine, J. Pile-Spellman, A theoretical model of selective cooling using intracarotid cold saline infusion in the human brain, Journal of Applied Physiology 102 (2006) 1329-1340.

[19] M. A. Neimark, A. A. Konstas, J. H. Choi, A. F.Laine, J. Pile-Spellman, Brain cooling maintenance with cooling cap following induction with intracarotid cold saline infusion: a quantitative model, Journal of Theoretical Biology 253 (2) (2008) 333-344.

[20] L. Zhu, C. Diao, Theoretical simulation of temperature distribuition in the brain during mild hypothermia treatment for brain injury, Medical Biological Engineering Computing 39 (6) (2001) 681-687.

[21] O.Ley, Y. Bayazitoglu, Effect of physiology on the temperature distribution of a layered head with external convection, International Journal of Heat and Mass Transfer 46 (17) (2003) 3233-3241.

[22] B. H. Dennis, R. C. Eberhart, G. S. Dulikravich, S. W. Radons, Finiteelement simulation of cooling of realistic 3 -d human head and neck, Journal of Biomechanical Engineering 125 (6) (2003) 832-840. 
[23] M. Zhu, J. J. Ackerman, A. L. Sukstanskii, D. A. Yablonskiy, How the body controls brain temperature: the temperature shielding effect of cerebral blood flow, Journal of Applied Physiology 101 (5) (2006) 1481-1488.

[24] C. J. Gordon, The therapeutic potential of regulated hypothermia, Emergency Medicine Journal 18 (2) (2001) 81-89.

[25] D. Fiala, G. Havenith, P. Brde, G. J. B. Kampmann, Utci-fiala multi-node model of human heat transfer and temperature regulation, International Journal of Biometeorology 56 (3) (2012) 429-441.

[26] M. Schwarz, M. W. Krueger, H. J. Busch, C. Benk, C. Heilmann, Modelbased assesment of tissue perfusion and temperature in deep hypothermic patients, IEEE Transactions on Biomedical Engineering 16 (3) (2010) $1577-$ 1586 .

[27] M. Schwarz, C. Heilmann, M. K. Krueger, U. Kiencke, Model based monitoring of hypothermic patients, Metrology and Measurement Systems 16 (2009) 443-455.

[28] A. Avolio, Multi-branched model of the human arterial system, Medical and Biological Engineering and Computing 18 (1980) 709-718.

[29] D. Fiala, Dynamic simulation of human heat transfer and thermal comfort, Phd thesis, De Montfort University, Leicester, UK (1998).

[30] B. R. Kingma, M. J. Vosselman, A. J. Frijns, A. A. V. Steenhoven, W. D. V. M. Lichtenbelt, Incorporationg neurophysiological concepts in mathematical thermoregulation models, International Journal of Biometeorology 58 (1) (2014) 87-99.

[31] J. E. Laszczyk, A. J. Nowak, The analysis of a newborns brain cooling process - measurements and CFD modelling, LAP LAMBERT Academic Publishing, Gliwice, 2015. 
[32] D.Fiala, K. J. Lomas, M. Stohrer, A computer model of human thermoregulation for a wide range of environmental conditions: the passive system, Journal of Applied Physiology 87 (5) (1999) 1957-1972.

[33] F. L. B. Ribeiro, I. A. Ferreira, Parallel implementation of the finite element method using compressed data structures, Computational Mechanics 41 (2007) 31-48.

[34] F. L. B. Ribeiro, A. L. G. A. Coutinho, Comparison between element, edge and compressed storage schemes for iterative solutions in finite element analyses, International Journal of Numerical Methods in Engineering 63 (4) (2005) 569-588.

[35] T. J. Hughes, The Finite Element Method - Linear Static and Dynamic Finites Element Analysis, Prentice-Hall International Editions, New Jersey, 1987.

[36] Y. Saad, Iterative Methods for Sparse Linear Systems, 2nd Edition, Society for Industrial Mathematics, Philadelphia, 2000.

[37] J. Laszczyk, A. Maczko, W. Walas, A. J. Nowak, The numerical modelling of the heat transfer processes within neonates body based on simplified geometric model, Information Technologies in Biomedicine, Lecture Notes in Computer Science 7339 (2012) 310-318.

[38] Z. Ostrowski, M. Rojczyk, J. Laszczyk, I. Szczygiel, J. Kaczmarczyk, A. J. Nowak, Infant care bed natural convection heat transfer coefficient - measurements and estimation, Przeglad Elektrotechniczny 5 (90) (2014) 122125.

[39] J. E. Laszczyk, A. J. Nowak, Computational modelling of neonates brain cooling, International Journal of Numerical Methods for Heat and Fluid Flow 26 (2016) 571-590. 
[40] J. Laszczyk, A. Maczko, W. Walas, A. J. Nowak, Inverse thermal analysis of the neonatal brain cooling process, International Journal of Numerical Methods for Heat and Fluid Flow 24 (2014) 949-968.

xxxiii 